\title{
TIME-PERIODIC SOLUTIONS OF THE VLASOV-POISSON-FOKKER-PLANCK SYSTEM
}

\author{
RENJUN DUAN AND SHUANGQIAN LIU
}

\begin{abstract}
In this note it is shown that the Vlasov-Poisson-Fokker-Planck system in the three-dimensional whole space driven by a time-periodic background profile near a positive constant state admits a time-periodic small-amplitude solution with the same period. The proof follows by the Serrin's method on the basis of the exponential time-decay property of the linearized system in the case of the constant background profile.
\end{abstract}

Dedicated to Professor Tai-Ping Liu on his 70th birthday

\section{INTRODUCTION}

Consider the Vlasov-Poisson-Fokker-Planck (VPFP for short) system

$$
\begin{aligned}
\partial_{t} F+\xi \cdot \nabla_{x} F+\nabla_{x} \Phi \cdot \nabla_{\xi} F & =\nabla_{\xi} \cdot\left(\nabla_{\xi} F+\xi F\right), \\
\Delta_{x} \Phi & =\int_{\mathbb{R}^{3}} F d \xi-\rho(t, x) .
\end{aligned}
$$

Here $F(t, x, \xi) \geq 0$ denotes the density distribution function of particles with position $x=\left(x_{1}, x_{2}, x_{3}\right) \in \mathbb{R}^{3}$ and velocity $\xi=\left(\xi_{1}, \xi_{2}, \xi_{3}\right) \in \mathbb{R}^{3}$ at time $t \in \mathbb{R}$, and $\Phi=\Phi(t, x)$ is the self-consistent potential function satisfying the Poisson equation with boundary condition

$$
\lim _{|x| \rightarrow \infty} \Phi(t, x)=0
$$

for all $t$. The background profile $\rho(t, x)$ is a given function which is periodic in time with the period $T>0$. Recall that the VPFP system is one of kinetic models in plasma physics for describing the motion of charged particles (e.g., electrons and ions) under the influence of the self-consistent electrostatic force and when collisions are governed by the Fokker-Planck operator; see [19] and reference therein.

In the paper we are concerned with the existence of time-periodic solutions with the same period $T$ for the VPFP system whenever $\rho(t, x)$ is smooth and sufficiently close to a positive constant state. With loss of generality we assume that $\rho(t, x)$ is around the unit constant. Define

$$
\phi(t, x)=\left(-\Delta_{x}\right)^{-1}(\rho(t, x)-1) .
$$

Date: July 4, 2015.

2010 Mathematics Subject Classification. 35Q84(primary), and 82D10(secondary).

Key words and phrases. Vlasov-Poisson-Fokker-Planck system, time-periodic solution, energy method, exponential time-decay. 
The above VPFP system can be also written as

$$
\begin{aligned}
\partial_{t} F+\xi \cdot \nabla_{x} F+\nabla_{x}(\Phi+\phi) \cdot \nabla_{\xi} F & =\nabla_{\xi} \cdot\left(\nabla_{\xi} F+\xi F\right), \\
\Delta_{x} \Phi & =\int_{\mathbb{R}^{3}} F d \xi-1,
\end{aligned}
$$

where for brevity we still have used the same notion $\Phi$ to denote the potential function. Define $M=(2 \pi)^{-3 / 2} \exp \left\{-|\xi|^{2} / 2\right\}$, and set the perturbation $f=f(t, x, \xi)$ by $F=M+M^{1 / 2} f$. The system is further reformulated as

$$
\begin{aligned}
& \partial_{t} f+\xi \cdot \nabla_{x} f+\nabla_{x}(\Phi+\phi) \cdot \nabla_{\xi} f \\
& \quad-\frac{1}{2} \xi \cdot \nabla_{x}(\Phi+\phi) f-\xi M^{1 / 2} \cdot \nabla_{x}(\Phi+\phi)=L f, \\
& \Delta_{x} \Phi=\int_{\mathbb{R}^{3}} M^{1 / 2} f d \xi .
\end{aligned}
$$

Here $L$ is the Fokker-Planck operator in the self-adjoint form given by

$$
L f=\frac{1}{M^{1 / 2}} \nabla_{\xi} \cdot\left[M \nabla_{\xi}\left(\frac{f}{M^{1 / 2}}\right)\right] .
$$

We introduce the function space

$$
\begin{aligned}
& X=\left\{f=f(x, \xi) \in L_{\xi}^{2}\left(H_{x}^{3}\right):\|f\|_{X}<\infty, M+M^{1 / 2} f \geq 0,\right. \\
&\left.\iint_{\mathbb{R}^{3} \times \mathbb{R}^{3}} M^{1 / 2} f(x, \xi) d \xi d x=0\right\}
\end{aligned}
$$

with the norm $\|\cdot\|_{X}$ defined by

$$
\|f\|_{X}^{2}=\|f\|_{L_{\xi}^{2}\left(H_{x}^{3}\right)}^{2}+\left\|\nabla_{x} \Phi^{f}\right\|_{H_{x}^{3}}^{2} .
$$

Here and in the sequel, for given $f(t, x, \xi), \Phi^{f}=\Phi^{f}(t, x)$ denotes

$$
\Phi^{f}(t, x)=-\frac{1}{4 \pi} \iint_{\mathbb{R}^{3} \times \mathbb{R}^{3}} \frac{M^{1 / 2} f(t, y, \xi)}{|x-y|} d \xi d y .
$$

The main result of the paper is stated as follows.

Theorem 1.1. Assume that $\phi(t, x)$ is time-periodic with period $T>0$. There are $\epsilon>0, C>0$ such that if

$$
\sup _{0 \leq t \leq T}\left\|\nabla_{x} \phi(t)\right\|_{H_{x}^{3}} \leq \epsilon
$$

then the reformulated VPFP system (1.3), (1.4) admits a unique time-periodic solution $f(t, x, \xi) \in X$ with the same period $T$ and

$$
\sup _{0 \leq t \leq T}\|f(t)\|_{X} \leq C \sup _{0 \leq t \leq T}\left\|\nabla_{x} \phi(t)\right\|_{H_{x}^{3} .} .
$$

In what follows we give some remarks on Theorem 1.1 related to its motivations. If $\phi \equiv 0$ corresponding to the case when the background profile $\rho$ is identical to a constant, the global Maxwellian $M$ is a trivial solution to the VPFP system (1.1), (1.2), and the global existence and the exponential time-decay of classical solutions near global Maxwellians have been established in [21] by the energy method (cf. [18]); see also [6, 12, 13, 17] for the study of similar problems with the FokkerPlanck operator. It is natural to ask what happens if the background profile depends on $t$ and $x$. 
When $\rho$ is a function of the only variable $x$ far from vacuum, one basic problem is to consider the existence and stability of the non-vacuum stationary solution. For the Vlasov-Poisson-Boltzmann system, it was studied in [16] provided that $\rho$ is smooth and sufficiently close to the positive constant state. Note that the Boltzmann collision operator is more degenerate than the Fokker-Planck and only the polynomial time-decay rate can be expected in the case of the whole space, cf. [14]. When $\rho$ depends on both $t$ and $x$, it is generally hard to obtain the global existence of classical solutions to the Vlasov-Poisson-type kinetic systems with collisions if $\rho(t, x)$ is only assumed to be bounded in all time, for instance, time-periodic in particular. This is because after linearization the time-dependent force must induce some inhomogeneous source terms which generally do not decay in time. However, in the case with small time-periodic driving forces, as long as the linear dynamics enjoys fast enough time-decay properties, it is still possible to obtain the existence and stability of time-periodic solutions with small amplitude. For the Boltzmann equation, it was studied in [26, 27] for the case with timeperiodic sources and in [15] for the case with given time-periodic forces. Note that it was additionally required in [15] that space dimensions have to be greater than or equal to five, and the physical case in dimensions three so far has remained open.

Motivated by those works mentioned above, we expect to consider the existence and stability of time-periodic solutions when collisions are replaced by the Fokker-Planck operator with stronger dissipation property. Indeed, the exponential time-decay property of the linearised VPFP system corresponding to constant background profiles plays a key role. To show Theorem 1.1, different from [15], we would use the Serrin's approach [25] (also cf. [22]), as it is more convenient to study the velocity-diffusive Fokker-Planck operator through pure energy estimates than using the Ukai's method in [26] in terms of the contraction mapping principle. It should be pointed out that the time-exponential stability of the obtained time-periodic solution under small perturbation can also be established as in [26], and its proof is omitted for brevity. Besides, it could be interesting to study the current problem but for the case of possibly large-amplitude background profiles, that is to remove the smallness assumption (1.5).

On the other hand, we mention that there have been a lot of works for the study of the VPFP system, for instance, both hypoelliptic [1, 3, 20] and hypocoercive $[4,9,10,11,20,28]$ properties, and the existence and large-time behaviour of solutions in different settings [2, 5, 7, 8, 23, 24, 29, 30].

Notations. Through the paper $C$ denotes a positive (generally large) constant and $\lambda$ a positive (generally small) constant, where both $C$ and $\lambda$ may take different values in different places. $A \sim B$ means $\lambda A \leq B \leq \frac{1}{\lambda} A$ for a generic constant $\lambda>0$. We use $\|\cdot\|$ to denote $L^{2}$ norm over $L_{x}^{2}$ or $L_{x, \xi}^{2}$ if no confusion arises, and use $\langle\cdot, \cdot \cdot\rangle$ to denote the inner product over $L_{x, \xi}^{2}$. For a multiple index $\alpha=\left(\alpha_{1}, \alpha_{2}, \alpha_{3}\right)$, we denote $\partial^{\alpha}=\partial_{x}^{\alpha}=\partial_{x_{1}}^{\alpha_{1}} \partial_{x_{2}}^{\alpha_{2}} \partial_{x_{3}}^{\alpha_{3}}$. The length of $\alpha$ is $|\alpha|=\alpha_{1}+\alpha_{2}+\alpha_{3}$.

\section{The Cauchy problem}

In this section we study the Cauchy problem on the reformulated VPFP system (1.3), (1.4) on $t>0$, supplemented with initial data

$$
f(0, x, \xi)=f_{0}(x, \xi) .
$$

The main result is stated as follows. 
Theorem 2.1. Assume that $f_{0} \in X, \nabla_{x} \phi \in C\left(0, \infty ; H_{x}^{3}\right)$ with

$$
\left\|f_{0}\right\|_{X}+\sup _{t \geq 0}\left\|\nabla_{x} \phi(t)\right\|_{H_{x}^{3}}
$$

sufficiently small. Then the Cauchy problem on the VPFP system (1.3), (1.4), (2.1) admits a unique solution $f(t, x, \xi) \in X$ with

$$
\sup _{t \geq 0}\|f(t)\|_{X} \leq C\left(\left\|f_{0}\right\|_{X}+\sup _{t \geq 0}\left\|\nabla_{x} \phi(t)\right\|_{H_{x}^{3}}\right) .
$$

The proof of Theorem 2.1 is based on the standard energy method with the help of the exponential time-decay property of the linearised system in the case when the background potential function $\phi$ is vanishing.

Let $\sigma(\xi)=1+|\xi|^{2}$. Denote $|\cdot|_{\sigma}$ by

$$
|f|_{\sigma}^{2}=\int_{\mathbb{R}^{3}}\left[\left|\nabla_{\xi} f\right|^{2}+\sigma(\xi)|f|^{2}\right] d \xi, \quad f=f(\xi) .
$$

Moreover, for $f=f(x, \xi),\|f\|_{\sigma}^{2}$ stands for the spatial integration of $|f(x, \cdot)|_{\sigma}^{2}$ over $\mathbb{R}^{3}$. Recall (cf. [12], for instance) that the operator $L$ satisfies that there is $\lambda_{0}>0$ such that

$$
-\int_{\mathbb{R}^{3}} f L f d \xi \geq \lambda_{0}\left|\left\{\mathbf{I}-\mathbf{P}_{0}\right\} f\right|_{\sigma}^{2}
$$

where $\mathbf{P}_{0} f=a^{f} M^{1 / 2}$, and $a^{f}(t, x)=\int_{\mathbb{R}^{3}} M^{1 / 2} f(t, x, \xi) d \xi$. We also introduce the velocity orthogonal projection $\mathbf{P}: L_{\xi}^{2} \rightarrow \operatorname{span}\left\{M^{1 / 2}, \xi M^{1 / 2}\right\}$ by $\mathbf{P}=\mathbf{P}_{0} \oplus \mathbf{P}_{1}$ with $\mathbf{P}_{1} f=b^{f} \cdot \xi M^{1 / 2}$ and $b^{f}(t, x)=\int_{\mathbb{R}^{3}} \xi M^{1 / 2} f(t, x, \xi) d \xi$.

Zero-order estimate: From (1.3) and (1.4), it is straightforward to obtain as in $[14,21]$

$$
\frac{1}{2} \frac{d}{d t}\left(\|f\|^{2}+\left\|\nabla_{x} \Phi^{f}\right\|^{2}\right)+\langle f,-L f\rangle=\left\langle\frac{1}{2} \xi \cdot \nabla_{x}\left(\Phi^{f}+\phi\right), f^{2}\right\rangle+\left\langle\xi M^{1 / 2} \cdot \nabla_{x} \phi, f\right\rangle .
$$

It then follows from (2.3) that

$$
\begin{aligned}
& \frac{1}{2} \frac{d}{d t}\left(\|f\|^{2}+\left\|\nabla_{x} \Phi^{f}\right\|^{2}\right)+\lambda_{0}\left\|\left\{\mathbf{I}-\mathbf{P}_{0}\right\} f\right\|_{\sigma}^{2} \\
& \leq\left\langle\frac{1}{2} \xi \cdot \nabla_{x}\left(\Phi^{f}+\phi\right), f^{2}\right\rangle+\left\langle\xi M^{1 / 2} \cdot \nabla_{x} \phi, f\right\rangle \\
& \leq C \sup _{x}\left\{\left|\nabla_{x} \Phi\right|,\left|\nabla_{x} \phi\right|\right\}\|f\|_{\sigma}^{2}+\eta\left\|b^{f}\right\|^{2}+C_{\eta}\left\|\nabla_{x} \phi\right\|^{2} \\
& \leq C\left\{\eta+\sup _{x}\left\{\left|\nabla_{x} \Phi\right|,\left|\nabla_{x} \phi\right|\right\}\|f\|_{\sigma}^{2}+C_{\eta}\left\|\nabla_{x} \phi\right\|^{2},\right.
\end{aligned}
$$

where $\eta>0$ is a constant to be chosen later on.

Higher-order estimate: Similarly as in the zero-order case, from (1.3) and (1.4), one has

$$
\begin{aligned}
& \frac{1}{2} \frac{d}{d t} \sum_{1 \leq|\alpha| \leq 3}\left(\left\|\partial^{\alpha} f\right\|^{2}+\left\|\partial^{\alpha} \nabla_{x} \Phi^{f}\right\|^{2}\right)+\sum_{1 \leq|\alpha| \leq 3}\left\langle\partial^{\alpha} f,-L \partial^{\alpha} f\right\rangle \\
& =-\sum_{1 \leq|\alpha| \leq 3}\left\langle\partial^{\alpha}\left[\nabla_{x}\left(\Phi^{f}+\phi\right) \cdot \nabla_{\xi} f\right], \partial^{\alpha} f\right\rangle+\sum_{1 \leq|\alpha| \leq 3}\left\langle\partial^{\alpha}\left[\frac{1}{2} \xi \cdot \nabla_{x}\left(\Phi^{f}+\phi\right) f\right], \partial^{\alpha} f\right\rangle \\
& \quad+\sum_{1 \leq|\alpha| \leq 3}\left\langle\xi M^{1 / 2} \cdot \partial^{\alpha} \nabla_{x} \phi, \partial^{\alpha} f\right\rangle .
\end{aligned}
$$


Let us introduce an equivalent energy functional

$$
\mathcal{E}(f) \sim\|f\|_{L_{\xi}^{2}\left(H_{x}^{3}\right)}^{2}+\left\|\nabla_{x} \Phi^{f}\right\|_{H_{x}^{3}}^{2}
$$

to be explicitly defined later on. Then the above estimate immediately gives

$$
\begin{aligned}
& \frac{1}{2} \frac{d}{d t} \sum_{1 \leq|\alpha| \leq 3}\left(\left\|\partial^{\alpha} f\right\|^{2}+\left\|\partial^{\alpha} \nabla_{x} \Phi^{f}\right\|^{2}\right)+\lambda_{0} \sum_{1 \leq|\alpha| \leq 3}\left\|\left\{\mathbf{I}-\mathbf{P}_{0}\right\} \partial^{\alpha} f\right\|_{\sigma}^{2} \\
& \leq C\left(\eta+\sqrt{\mathcal{E}(f)}+\left\|\nabla_{x} \phi\right\|_{H^{3}}\right) \sum_{|\alpha| \leq 3}\left(\left\|\partial^{\alpha} f\right\|_{\sigma}^{2}+\left\|\partial^{\alpha} \nabla_{x} \Phi^{f}\right\|^{2}\right) \\
& \quad+C_{\eta}\left\|\nabla_{x} \phi\right\|_{H^{3}}^{2} .
\end{aligned}
$$

Dissipation of $a^{f}$ and $\nabla_{x} \Phi^{f}$ : As in [12], applying $\mathbf{P}$ to (1.3), it follows together with (1.4) that

$$
\begin{aligned}
& \partial_{t} a^{f}+\nabla_{x} \cdot b^{f}=0, \\
& \partial_{t} b^{f}+\nabla_{x} a^{f}+\nabla_{x} \cdot \Gamma(\{\mathbf{I}-\mathbf{P}\} f)=-b^{f}+\left(1+a^{f}\right) \nabla_{x}\left(\Phi^{f}+\phi\right), \\
& \Delta_{x} \Phi^{f}=a^{f},
\end{aligned}
$$

where $\Gamma=\left(\Gamma_{i j}\right)_{1 \leq i, j \leq 3}$ is the moment functional defined by

$$
\Gamma_{i j}(f)=\int_{\mathbb{R}^{3}}\left(\xi_{i} \xi_{j}-1\right) M^{1 / 2} f d \xi .
$$

Let $|\alpha| \leq 3$. Applying $\partial^{\alpha}$ to (2.8), taking the inner product of the resulting equation with $\partial^{\alpha} \nabla_{x} \Phi^{f}$ over $\mathbb{R}_{x}^{3}$ and using (2.9), we obtain

$$
\begin{aligned}
& \left\|\partial^{\alpha} \nabla_{x} \Phi^{f}\right\|^{2}+\left\|\partial^{\alpha} a^{f}\right\|^{2} \\
& =\int_{\mathbb{R}^{3}} \partial_{t} \partial^{\alpha} b^{f} \cdot \partial^{\alpha} \nabla_{x} \Phi^{f} d x+\int_{\mathbb{R}^{3}} \nabla_{x} \cdot \Gamma\left(\{\mathbf{I}-\mathbf{P}\} \partial^{\alpha} f\right) \cdot \partial^{\alpha} \nabla_{x} \Phi^{f} d x \\
& \quad+\int_{\mathbb{R}^{3}} \partial^{\alpha} b^{f} \cdot \partial^{\alpha} \nabla_{x} \Phi^{f} d x-\int_{\mathbb{R}^{3}} \partial^{\alpha}\left[a^{f} \nabla_{x}\left(\Phi^{f}+\phi\right)\right] \cdot \partial^{\alpha} \nabla_{x} \Phi^{f} d x .
\end{aligned}
$$

Denote the right-hand terms by $I_{i}(1 \leq i \leq 4)$. For $I_{1}$, it is given by

$$
I_{1}=\frac{d}{d t} \int_{\mathbb{R}^{3}} \partial^{\alpha} b^{f} \cdot \partial^{\alpha} \nabla_{x} \Phi^{f} d x-\int_{\mathbb{R}^{3}} \partial^{\alpha} b^{f} \cdot \partial^{\alpha} \nabla_{x} \partial_{t} \Phi^{f} d x,
$$

where one can use (2.7) to further compute

$$
\begin{aligned}
-\int_{\mathbb{R}^{3}} \partial^{\alpha} b^{f} \cdot \partial^{\alpha} \nabla_{x} \partial_{t} \Phi^{f} d x & =\int_{\mathbb{R}^{3}} \partial^{\alpha} \nabla_{x} \cdot b^{f} \partial^{\alpha} \Delta_{x}^{-1} \partial_{t} a^{f} d x \\
& =-\int_{\mathbb{R}^{3}} \partial^{\alpha} \nabla_{x} \cdot b^{f} \Delta_{x}^{-1} \partial^{\alpha} \nabla_{x} \cdot b^{f} d x \\
& =\int_{\mathbb{R}^{3}}\left|\nabla_{x} \Delta_{x}^{-1} \partial^{\alpha} \nabla_{x} \cdot b^{f}\right|^{2} d x .
\end{aligned}
$$

As the operator $\nabla_{x} \Delta_{x}^{-1} \nabla_{x}$. is bounded from $L^{p}$ to $L^{p}(1<p<\infty)$, it then follows that

and hence

$$
\int_{\mathbb{R}^{3}}\left|\nabla_{x} \Delta_{x}^{-1} \partial^{\alpha} \nabla_{x} \cdot b^{f}\right|^{2} d x \leq C\left\|\partial^{\alpha} b^{f}\right\|^{2},
$$

$$
I_{1} \leq \frac{d}{d t} \int_{\mathbb{R}^{3}} \partial^{\alpha} b^{f} \cdot \partial^{\alpha} \nabla_{x} \Phi^{f} d x+C\left\|\partial^{\alpha} b^{f}\right\|^{2} .
$$


For $I_{2}$, from taking integration by part, using (2.9) and noticing again the boundedness of $\partial_{x_{i}} \partial_{x_{j}} \Delta_{x}^{-1}$ on $L^{2}$, one has

$$
\begin{aligned}
I_{2} & =\sum_{i j} \int_{\mathbb{R}^{3}} \partial_{x_{j}} \Gamma_{i j}\left(\{\mathbf{I}-\mathbf{P}\} \partial^{\alpha} f\right) \partial^{\alpha} \partial_{x_{i}} \Phi^{f} d x \\
& =-\sum_{i j} \int_{\mathbb{R}^{3}} \Gamma_{i j}\left(\{\mathbf{I}-\mathbf{P}\} \partial^{\alpha} f\right) \partial^{\alpha} \partial_{x_{i}} \partial_{x_{j}} \Delta_{x}^{-1} a^{f} d x \\
& \leq \eta\left\|\partial^{\alpha} \partial_{x_{i}} \partial_{x_{j}} \Delta_{x}^{-1} a^{f}\right\|^{2}+C_{\eta}\left\|\Gamma\left(\{\mathbf{I}-\mathbf{P}\} \partial^{\alpha} f\right)\right\|^{2} \\
& \leq C \eta\left\|\partial^{\alpha} a^{f}\right\|^{2}+C_{\eta}\left\|\{\mathbf{I}-\mathbf{P}\} \partial^{\alpha} f\right\|^{2} .
\end{aligned}
$$

For the rest two terms, it is straightforward to obtain

$$
I_{3} \leq \eta\left\|\partial^{\alpha} \nabla_{x} \Phi^{f}\right\|^{2}+C_{\eta}\left\|\partial^{\alpha} b^{f}\right\|^{2},
$$

and

$$
\begin{aligned}
I_{4} & =-\int_{\mathbb{R}^{3}} \partial^{\alpha}\left[a^{f} \nabla_{x}\left(\Phi^{f}+\phi\right)\right] \cdot \partial^{\alpha} \nabla_{x} \Phi^{f} d x \\
& \leq C\left\|a^{f}\right\|_{H^{3}}\left\|\nabla_{x} \Phi^{f}\right\|^{2}+\left\|\nabla_{x} \phi\right\|_{H^{3}}\left\|a^{f}\right\|_{H^{3}}\left\|\nabla_{x} \Phi^{f}\right\|_{H^{3}} \\
& \leq\left(\left\|a^{f}\right\|_{H^{3}}+\left\|\nabla_{x} \phi\right\|_{H^{3}}\right)\left(\left\|a^{f}\right\|_{H^{3}}^{2}+\left\|\nabla_{x} \Phi^{f}\right\|_{H^{3}}^{2}\right) .
\end{aligned}
$$

Plugging the above estimates into (2.10), using $\left|\partial^{\alpha} b^{f}\right| \leq C\left\|\left\{\mathbf{I}-\mathbf{P}_{0}\right\} \partial^{\alpha} f\right\|_{L_{\xi}^{2}}$ and then taking the sum over $|\alpha| \leq 3$, it holds that

$$
\begin{aligned}
& -\frac{d}{d t} \sum_{|\alpha| \leq 3} \int_{\mathbb{R}^{3}} \partial^{\alpha} b^{f} \cdot \partial^{\alpha} \nabla_{x} \Phi^{f} d x+\lambda\left(\left\|\nabla_{x} \Phi^{f}\right\|_{H^{3}}^{2}+\left\|a^{f}\right\|_{H^{3}}^{2}\right) \\
& \leq C\left\|\left\{\mathbf{I}-\mathbf{P}_{0}\right\} f\right\|_{L_{\xi}^{2}\left(H_{x}^{3}\right)}^{2}+C\left(\left\|a^{f}\right\|_{H^{3}}+\left\|\nabla_{x} \phi\right\|_{H^{3}}\right)\left(\left\|a^{f}\right\|_{H^{3}}^{2}+\left\|\nabla_{x} \Phi^{f}\right\|_{H^{3}}^{2}\right) .
\end{aligned}
$$

This completes the estimate on the dissipation of $a^{f}$ and $\nabla_{x} \Phi^{f}$.

We now define

$$
\mathcal{E}(f)=\|f\|_{L_{\xi}^{2}\left(H_{x}^{3}\right)}^{2}+\left\|\nabla_{x} \Phi^{f}\right\|_{H_{x}^{3}}^{2}-\kappa \sum_{|\alpha| \leq 3} \int_{\mathbb{R}^{3}} \partial^{\alpha} b^{f} \cdot \partial^{\alpha} \nabla_{x} \Phi^{f} d x,
$$

with the constant $\kappa>0$ small enough. Note that $\mathcal{E}(f)$ satisfies $(2.5)$ as long as $\kappa>0$ is small enough. By taking $\kappa>0$ to be further small enough, it follows from $(2.4),(2.6)$ and $(2.11)$ that

$$
\begin{aligned}
\frac{d}{d t} \mathcal{E}(f)+ & \lambda \sum_{|\alpha| \leq 3}\left\|\left\{\mathbf{I}-\mathbf{P}_{0}\right\} \partial^{\alpha} f\right\|_{\sigma}^{2}+\lambda\left(\left\|\nabla_{x} \Phi^{f}\right\|_{H^{3}}^{2}+\left\|a^{f}\right\|_{H^{3}}^{2}\right) \\
\leq C\left(\eta+\sqrt{\mathcal{E}(f)}+\left\|\nabla_{x} \phi\right\|_{H^{3}}\right) \sum_{|\alpha| \leq 3}\left(\left\|\partial^{\alpha} f\right\|_{\sigma}^{2}+\left\|\partial^{\alpha} \nabla_{x} \Phi^{f}\right\|^{2}\right) & +C_{\eta}\left\|\nabla_{x} \phi\right\|_{H^{3}}^{2} .
\end{aligned}
$$


Notice that $\mathcal{E}(f) \sim\|f\|_{X}^{2}$ and

$$
\begin{aligned}
\mathcal{E}(f) & \leq C\left(\|f\|_{L_{\xi}^{2}\left(H_{x}^{3}\right)}^{2}+\left\|\nabla_{x} \Phi^{f}\right\|_{H_{x}^{3}}^{2}\right) \\
& \leq C \sum_{|\alpha| \leq 3}\left(\left\|\partial^{\alpha} f\right\|_{\sigma}^{2}+\left\|\partial^{\alpha} \nabla_{x} \Phi^{f}\right\|^{2}\right) \\
& \leq C \sum_{|\alpha| \leq 3}\left\|\left\{\mathbf{I}-\mathbf{P}_{0}\right\} \partial^{\alpha} f\right\|_{\sigma}^{2}+C\left(\left\|\nabla_{x} \Phi^{f}\right\|_{H^{3}}^{2}+\left\|a^{f}\right\|_{H^{3}}^{2}\right) .
\end{aligned}
$$

Fix $\eta>0$ to be a small constant. Therefore, by the standard continuity argument, whenever $\left\|f_{0}\right\|_{X}$ and $\sup _{t}\left\|\nabla_{x} \phi(t)\right\|_{H^{3}}$ are sufficiently small, it follows from (2.13) that

$$
\frac{d}{d t} \mathcal{E}(f)+\lambda \mathcal{E}(f) \leq C\left\|\nabla_{x} \phi\right\|_{H^{3}}^{2},
$$

which by Gronwall's inequality implies

$$
\begin{aligned}
\mathcal{E}(f(t)) \leq \mathcal{E}\left(f_{0}\right) e^{-\lambda t}+C \int_{0}^{t} e^{-\lambda(t-s)} & \|\nabla \phi(s)\|_{H^{3}}^{2} d s \\
& \leq \mathcal{E}\left(f_{0}\right) e^{-\lambda t}+C\left(\sup _{t \geq 0}\|\nabla \phi(t)\|_{H^{3}}\right)^{2},
\end{aligned}
$$

and hence

$$
\|f(t)\|_{X} \leq C\left(\left\|f_{0}\right\|_{X}+\sup _{t \geq 0}\|\nabla \phi(t)\|_{H^{3}}\right)
$$

for all $t \geq 0$. This closes the a priori estimate as $\left\|f_{0}\right\|_{X}$ and $\sup _{t}\left\|\nabla_{x} \phi(t)\right\|_{H^{3}}$ are chosen to be small enough. The proof for the local existence of solutions in $X$ is omitted for brevity; see [6], for instance. Therefore, Theorem 2.1 is proved.

\section{TIME-PERIODiC SOLUTIONS}

In this section we shall use the Serrin's approach (cf. [22, 25]) to prove Theorem 1.1. To the end, we assume that $\phi(t, x)$ is periodic in time with period $T>0$, and

$$
\delta_{\phi}:=\sup _{0 \leq t \leq T}\left\|\nabla_{x} \phi(t)\right\|_{H^{3}}
$$

is sufficiently small.

Step 1: Find the special initial data. Let $f(t, \cdot, \cdot) \in X(t \geq 0)$ be the solution obtained in Theorem 2.1 by solving the Cauchy problem (1.3) and (1.4) corresponding to initial data $f_{0}(x, \xi)$ with $\left\|f_{0}\right\|_{X} \leq \delta_{0}$ with $\delta_{0}>0$ small enough. Take integers $m \geq k \geq 1$, and define

$$
g(t, x, \xi)=f(t+(m-k) T, x, \xi) .
$$

As $\phi(t, x)$ is time-periodic with period $T$, it is direct to see that $g(t, x, \xi)$ solves the Cauchy problem on the same VPFP system

$$
\begin{aligned}
& \partial_{t} g+\xi \cdot \nabla_{x} g+\nabla_{x}\left(\Phi^{g}+\phi\right) \cdot \nabla_{\xi} g \\
& \quad-\frac{1}{2} \xi \cdot \nabla_{x}\left(\Phi^{g}+\phi\right) g-\xi M^{1 / 2} \cdot \nabla_{x}\left(\Phi^{g}+\phi\right)=L g, \\
& \Delta_{x} \Phi^{g}=\int_{\mathbb{R}^{3}} M^{1 / 2} g d \xi
\end{aligned}
$$

with initial data $g(0, x, \xi)=f((m-k) T, x, \xi)$. We define

$$
h(t, x, \xi)=g(t, x, \xi)-f(t, x, \xi), \quad \Phi^{h}(t, x)=\Phi^{g}(t, x)-\Phi^{f}(t, x) .
$$


After taking the difference of two systems of $g$ and $f, h(t, x, \xi)$ satisfies

$$
\begin{aligned}
& \partial_{t} h+\xi \cdot \nabla_{x} h+\nabla_{x}\left(\Phi^{h}+\phi\right) \cdot \nabla_{\xi} h \\
& -\frac{1}{2} \xi \cdot \nabla_{x}\left(\Phi^{h}+\phi\right) h-\xi M^{1 / 2} \cdot \nabla_{x} \Phi^{h}=L h+R, \\
& \Delta_{x} \Phi^{h}=\int_{\mathbb{R}^{3}} M^{1 / 2} h d \xi
\end{aligned}
$$

where $R$ is denoted by

$$
R=\frac{1}{2} \xi \cdot \nabla_{x} \Phi^{f} h-\nabla_{x} \Phi^{f} \cdot \nabla_{\xi} h+\frac{1}{2} \xi \cdot \nabla_{x} \Phi^{h} f-\nabla_{x} \Phi^{h} \cdot \nabla_{\xi} f .
$$

Notice that $h$ satisfies the same system as $f$ except that the remaining term $R$ linear in $h$ appears and the time-periodic driving inhomogeneous source term $\xi M^{1 / 2} \cdot \nabla_{x} \phi$ is vanishing. Based on this observation, one can repeat the similar energy estimates as done for the Cauchy problem on $f$ in the past section. First of all, we claim that

$$
\sum_{|\alpha| \leq 3}\left\langle\partial^{\alpha} R, \partial^{\alpha} h\right\rangle \leq C\left(\delta_{0}+\delta_{\phi}\right)\left(\sum_{|\alpha| \leq 3}\left\|\partial^{\alpha} h\right\|_{\sigma}^{2}+\left\|\nabla_{x} \Phi^{h}\right\|_{H^{3}}^{2}\right) .
$$

Indeed, for each $\alpha$ with $|\alpha| \leq 3$,

$$
\begin{aligned}
&\left\langle\partial^{\alpha} R, \partial^{\alpha} h\right\rangle \\
&=\left\langle\partial^{\alpha}\left(\frac{1}{2} \xi \cdot \nabla_{x} \Phi^{f} h-\nabla_{x} \Phi^{f} \cdot \nabla_{\xi} h\right), \partial^{\alpha} h\right\rangle+\left\langle\partial^{\alpha}\left(\frac{1}{2} \xi \cdot \nabla_{x} \Phi^{h} f\right), \partial^{\alpha} h\right\rangle \\
&+\left\langle\partial^{\alpha}\left(\nabla_{x} \Phi^{h} f\right), \partial^{\alpha} \nabla_{\xi} h\right\rangle \\
& \leq C\left\|\nabla_{x} \Phi^{f}\right\|_{H^{3}} \sum_{|\alpha| \leq 3}\left\|\partial^{\alpha} h\right\|_{\sigma}^{2}+C\|f\|_{L_{\xi}^{2}\left(H_{x}^{3}\right)}\left(\sum_{|\alpha| \leq 3}\left\|\partial^{\alpha} h\right\|_{\sigma}^{2}+\left\|\nabla_{x} \Phi^{h}\right\|_{H^{3}}^{2}\right),
\end{aligned}
$$

where we have taken the integration by part in $\xi$ variable in the last term of $R$. Recall that from Theorem 2.1,

$$
\|f\|_{L_{\xi}^{2}\left(H_{x}^{3}\right)}+\left\|\nabla_{x} \Phi^{f}\right\|_{H^{3}}=\|f\|_{X} \leq C\left(\left\|f_{0}\right\|_{X}+\sup _{t \geq 0}\left\|\nabla_{x} \phi(t)\right\|_{H_{x}^{3}}\right) \leq C\left(\delta_{0}+\delta_{\phi}\right) .
$$

Therefore (3.5) holds true. Then, by modifying the proof of (2.4) and (2.6), one can obtain

$$
\begin{gathered}
\frac{1}{2} \frac{d}{d t}\left(\|h\|^{2}+\left\|\nabla_{x} \Phi^{h}\right\|^{2}\right)+\lambda_{0}\left\|\left\{\mathbf{I}-\mathbf{P}_{0}\right\} h\right\|_{\sigma}^{2} \\
\leq C \sup _{x}\left\{\left|\nabla_{x} \Phi^{h}\right|,\left|\nabla_{x} \phi\right|\right\}\|h\|_{\sigma}^{2}+\langle R, h\rangle \\
\leq C\left(\delta_{0}+\delta_{\phi}\right)\left(\sum_{|\alpha| \leq 3}\left\|\partial^{\alpha} h\right\|_{\sigma}^{2}+\left\|\nabla_{x} \Phi^{h}\right\|_{H^{3}}^{2}\right),
\end{gathered}
$$

and

$$
\begin{gathered}
\frac{1}{2} \frac{d}{d t} \sum_{1 \leq|\alpha| \leq 3}\left(\left\|\partial^{\alpha} h\right\|^{2}+\left\|\partial^{\alpha} \nabla_{x} \Phi^{h}\right\|^{2}\right)+\lambda_{0} \sum_{1 \leq|\alpha| \leq 3}\left\|\left\{\mathbf{I}-\mathbf{P}_{0}\right\} \partial^{\alpha} h\right\|_{\sigma}^{2} \\
\leq C\left(\sqrt{\mathcal{E}(h)}+\left\|\nabla_{x} \phi\right\|_{H^{3}}\right) \sum_{|\alpha| \leq 3}\left(\left\|\partial^{\alpha} h\right\|_{\sigma}^{2}+\left\|\partial^{\alpha} \nabla_{x} \Phi^{h}\right\|^{2}\right)+\sum_{1 \leq|\alpha| \leq 3}\left\langle\partial^{\alpha} R, \partial^{\alpha} h\right\rangle \\
\leq C\left(\delta_{0}+\delta_{\phi}\right)\left(\sum_{|\alpha| \leq 3}\left\|\partial^{\alpha} h\right\|_{\sigma}^{2}+\left\|\nabla_{x} \Phi^{h}\right\|_{H^{3}}^{2}\right) .
\end{gathered}
$$


As before, to obtain the dissipation of $a^{h}$ and $\nabla_{x} \Phi^{h}$, we deduce from the system of $h(3.3)$ and (3.4) that

$$
\begin{aligned}
& \partial_{t} a^{h}+\nabla_{x} \cdot b^{h}=0, \\
& \partial_{t} b^{h}+\nabla_{x} a^{h}+\nabla_{x} \cdot \Gamma(\{\mathbf{I}-\mathbf{P}\} h) \\
& =-b^{h}+\nabla_{x} \Phi^{h}+a^{h} \nabla_{x} \phi+a^{h} \nabla_{x} \Phi^{g}+a^{f} \nabla_{x} \Phi^{h}, \\
& \Delta_{x} \Phi^{h}=a^{h} .
\end{aligned}
$$

By repeating the similar computations as for obtaining (2.11), one has

$$
\begin{aligned}
& -\frac{d}{d t} \sum_{|\alpha| \leq 3} \int_{\mathbb{R}^{3}} \partial^{\alpha} b^{h} \cdot \partial^{\alpha} \nabla_{x} \Phi^{h} d x+\lambda\left(\left\|\nabla_{x} \Phi^{h}\right\|_{H^{3}}^{2}+\left\|a^{h}\right\|_{H^{3}}^{2}\right) \\
& \quad \leq C\left\|\left\{\mathbf{I}-\mathbf{P}_{0}\right\} h\right\|_{L_{\xi}^{2}\left(H_{x}^{3}\right)}^{2}+C\left(\delta_{0}+\delta_{\phi}\right)\left(\left\|a^{h}\right\|_{H^{3}}^{2}+\left\|\nabla_{x} \Phi^{h}\right\|_{H^{3}}^{2}\right) .
\end{aligned}
$$

Then, recall (2.12), and by combing (3.6), (3.7) and (3.8) and using smallness of $\delta_{0}$ and $\delta_{\phi}$, it holds that

which implies

$$
\frac{d}{d t} \mathcal{E}(h)+\lambda \mathcal{E}(h) \leq 0,
$$

$$
\|h(t)\|_{X} \leq C \mathcal{E}(h(t)) \leq C \mathcal{E}(h(0)) e^{-\lambda t} \leq C\|h(0)\|_{X} e^{-\lambda t},
$$

for all $t \geq 0$. Recall (3.1) and (3.2). Using (2.2), the above inequality yields

$$
\begin{aligned}
\|f(t+(m-k) T)-f(t)\|_{X} & \leq C\|f((m-k) T)-f(0)\|_{X} e^{-\lambda t} \\
& \leq C\left(\|f((m-k) T)\|_{X}+\|f(0)\|_{X}\right) e^{-\lambda t} \\
& \leq C\left(\|f(0)\|_{X}+\sup _{t \geq 0}\left\|\nabla_{x} \phi(t)\right\|_{H_{x}^{3}}\right) e^{-\lambda t} \\
& \leq C\left(\delta_{0}+\delta_{\phi}\right) e^{-\lambda t} .
\end{aligned}
$$

Taking $t=k T$, one has

$$
\|f(m T)-f(k T)\|_{X} \leq C\left(\delta_{0}+\delta_{\phi}\right) e^{-\lambda k T},
$$

for all integers $m \geq k \geq 1$. As $e^{-\lambda k T} \rightarrow 0$ as $k \rightarrow \infty$, it shows that $\{f(k T, \cdot, \cdot)\}_{k \geq 1} \subset$ $X$ is a Cauchy sequence with respect to the norm $\|\cdot\|_{X}$, and its limit function is denoted by $f_{0}^{*}=f_{0}^{*}(x, \xi) \in X$ which satisfies

$$
\left\|f_{0}^{*}\right\|_{X} \leq C\left(\delta_{0}+\delta_{\phi}\right) \text {. }
$$

Step 2: Solve the Cauchy problem with initial data $f_{0}^{*}$. As both $\delta_{0}$ and $\delta_{\phi}$ are small enough, so is $\left\|f_{0}^{*}\right\|_{X}$ due to (3.10). Thus, applying Theorem 2.1, one can find $f^{*}(t, x, \xi)$ which is the solution to the Cauchy problem (1.3), (1.4) with initial data given by $f_{0}^{*}(x, \xi) \in X$. For an integer $n \geq 1$, we define

$$
\tilde{h}(t, x, \xi)=f(t+n T, x, \xi)-f^{*}(t, x, \xi),
$$

where $f(t, x, \xi)$ is the solution used for obtaining $f_{0}^{*}(x, \xi)$ in the previous step. By applying the same estimate as $h$ in (3.9) to $\tilde{h}(t, x, \xi)$, it holds that

$$
\left\|f(t+n T)-f^{*}(t)\right\|_{X} \leq C\left\|f(n T)-f^{*}(0)\right\|_{X} e^{-\lambda t},
$$

for all $t \geq 0$. Letting $t=T$ in the above inequality, one has

$$
\left\|f((n+1) T)-f^{*}(T)\right\|_{X} \leq C\left\|f(n T)-f_{0}^{*}\right\|_{X},
$$


which by further taking $n \rightarrow \infty$, implies $\left\|f_{0}^{*}-f^{*}(T)\right\|_{X}=0$, namely

$$
\left\|f^{*}(0)-f^{*}(T)\right\|_{X}=0 .
$$

Therefore it shows that $f^{*}(t, x, \xi) \in X$ is periodic in time with the period $T$. Notice that $f^{*}(t, x, \xi)$ satisfies the system (1.3), (1.4) on all $t \geq 0$, particularly on $0 \leq t \leq T$. It remains to prove (1.6). In fact, as for obtaining (2.14), it holds that

$$
\mathcal{E}\left(f^{*}(t)\right) \leq C \mathcal{E}\left(f_{0}^{*}\right) e^{-\lambda t}+C\left(\sup _{0 \geq 0}\|\nabla \phi(t)\|_{H^{3}}\right)^{2},
$$

that is,

$$
\left\|f^{*}(t)\right\|_{X} \leq C\left\|f_{0}^{*}\right\|_{X} e^{-\lambda t}+C \sup _{0 \leq t \leq T}\|\nabla \phi(t)\|_{H^{3}}
$$

for all $t \geq 0$. Then, for $0 \leq t \leq T$,

$$
\left\|f^{*}(t)\right\|_{X}=\left\|f^{*}(t+n T)\right\|_{X} \leq C\left\|f_{0}^{*}\right\|_{X} e^{-\lambda n T}+C \sup _{0 \leq t \leq T}\|\nabla \phi(t)\|_{H^{3}} .
$$

Hence (1.6) follows by taking $n \rightarrow \infty$. The proof of Theorem 1.1 is complete.

Acknowledgements: RJD was supported by the General Research Fund (Project No. 409913) from RGC of Hong Kong. SQL was supported by grants from the National Natural Science Foundation of China \#11101188 and \#11271160.

\section{REFERENCES}

[1] F. Bouchut, Hypoelliptic regularity in kinetic equations, J. Math. Pures Appl. (9) 81 (2002), $1135-1159$.

[2] F. Bouchut, Existence and uniqueness of a global smooth solution for the Vlasov-PoissonFokker-Planck system in three dimensions, J. Funct. Anal. 111 (1993), 239-258.

[3] F. Bouchut, Smoothing effect for the non-linear Vlasov-Poisson-Fokker-Planck system, J. Differential Equations 122 (1995), 225-238.

[4] F. Bouchut and J. Dolbeault, On long time asymptotics of the Vlasov-Fokker-Planck equation and of the Vlasov-Poisson-Fokker-Planck system with Coulombic and Newtonian potentials, Differential Integral Equations 8 (1995), 487-514.

[5] A. Carpio, Long-time behaviour for solutions of the Vlasov-Poisson-Fokker-Planck equation, Math. Methods Appl. Sci. 21 (1998), 985-1014.

[6] J. Carrillo, R. Duan and A. Moussa, Global classical solutions close to equilibrium to the Vlasov-Fokker-Planck-Euler system, Kinet. Relat. Models 4 (2011), 227-258.

[7] J. A. Carrillo and J. Soler, On the initial value problem for the Vlasov-Poisson-Fokker-Planck system with initial data in Lp spaces, Math. Methods Appl. Sci. 18 (1995), 825-839.

[8] J. A. Carrillo, J. Soler and J. L. Vazquez, Asymptotic behaviour and self-similarity for the three-dimensional Vlasov-Poisson-Fokker-Planck system, J. Ftinct. Anal. 141 (1996), 99-132.

[9] L. Desvillettes and C. Villani, On the trend to global equilibrium in spatially inhomogeneous entropy-dissipating systems: The linear Fokker-Planck equation, Comm. Pure Appl. Math. 54 (2001), 1-42.

[10] L. Desvillettes and C. Villani, On the trend to global equilibrium for spatially inhomogeneous kinetic systems: The Boltzmann equation, Invent. Math. 159 (2005), 245-316.

[11] R.-J. Duan, Hypocoercivity of linear degenerately dissipative kinetic equations, Nonlinearity 24 (2011), no. 8, 2165-2189.

[12] R.-J. Duan, M. Fornasier and G. Toscani, A kinetic flocking model with diffusion, Comm. Math. Phys. 300 (2010), no. 1, 95-145.

[13] R.-J. Duan and S.Q. Liu, Cauchy problem on the Vlasov-Fokker-Planck equation coupled with the compressible Euler equations through the friction force, Kinet. Relat. Models 6 (2013), no. 4, 687-700.

[14] R.-J. Duan and R. Strain, Optimal time decay of the Vlasov-Poisson-Boltzmann system in $\mathbb{R}^{3}$, Arch. Ration. Mech. Anal. 199 (2011), 291-328. 
[15] R.-J. Duan, S. Ukai, T. Yang, H.J. Zhao, Optimal decay estimates on the linearized Boltzmann equation with time dependent force and their applications, Comm. Math. Phys. 277 (2008), no. 1, 189-236.

[16] R.-J. Duan and T. Yang, Stability of the one-species Vlasov-Poisson-Boltzmann system, SIAM J. Math. Anal. 41 (2009/10), no. 6, 2353-2387.

[17] R. Esposito, Y. Guo and R. Marra, Stability of the front under a Vlasov-Fokker-Planck dynamics, Arch. Ration. Mech. Anal. 195 (2010), 75-116.

[18] Y. Guo, The Vlasov-Poisson-Boltzmann system near Maxwellians, Comm. Pure Appl. Math. 55 (2002), 1104-1135.

[19] Y. Guo, The Vlasov-Poisson-Landau system in a periodic box, J. Amer. Math. Soc. 25 (2012) 759-812.

[20] F. Hérau and F. Nier, Isotropic hypoellipticity and trend to equilibrium for the Fokker-Planck equation with a high-degree potential, Arch. Ration. Mech. Anal. 171 (2004), 151-218.

[21] H.J. Hwang and J. Jang, On the Vlasov-Poisson-Fokker-Planck equation near Maxwellian, Discrete Contin. Dyn. Syst. Ser. B 18 (2013), 681-691.

[22] P. Maremonti, Existence and stability of time-periodic solutions to the Navier-Stokes equations in the whole space, Nonlinearity 4 (1991), 503-529.

[23] K. Ono and W. A. Strauss, Regular solutions of the Vlasov-Poisson-Fokker-Planck system, Discrete Contin. Dynam. Systems 6 (2000), 751-772.

[24] G. Rein and J. Weckler, Generic global classical solutions of the Vlasov-Fokker-Planck-Poisson system in three dimensions, J. Differential Equations 99 (1992), 59-77.

[25] J. Serrin, A note on the existence of periodic solutions of the Navier-Stokes equations, Arch. Rational Mech. Anal. 3 (1959), 120-122.

[26] S. Ukai, Time-periodic solutions of the Boltzmann equation, Discrete Cont. Dyn. Syst. 14 (2006), 579-596.

[27] S. Ukai and T. Yang, The Boltzmann equation in the space $L^{2} \cap L_{\beta}^{\infty}$ : Global and time-periodic solutions, Anal. Appl. 4 (2006), 263-310

[28] C. Villani, Hypocoercivity, Mem. Amer. Math. Soc. 202 (2009), iv+141 pp.

[29] H. D. Victory, On the existence of global weak solutions for Vlasov-Poisson-Fokker-Planck systems, J. Math. Anal. Appl. 160 (1991), 525-555.

[30] H. D. Victory, Jr. and B. P. O'Dwyer, On classical solutions of Vlasov-Poisson Fokker-Planck systems, Indiana Univ. Math. J. 39 (1990), 105-156.

(RJD) Department of Mathematics, The Chinese University of Hong Kong, Shatin, Hong Kong, P.R. China

E-mail address: rjduan@math.cuhk.edu.hk

(SQL) Department of Mathematics, Jinan Unviersity, Guangdong, P.R. China

E-mail address: tsqliu@jnu.edu.cn 